\title{
Exploring the obscure profiles of pharmacological binding sites on voltage-gated sodium channels by BmK neurotoxins
}

\author{
Zhi-Rui Liu, Pin Ye, Yong-Hua Ji ${ }^{\bowtie}$ \\ Laboratory of Neuropharmacology and Neurotoxicology, Shanghai University, Shanghai 200444, China \\ $\triangle$ Correspondence: yhji@staff.shu.edu.cn \\ Received May 26, 2011 Accepted June 8, 2011
}

\section{ABSTRACT}

Diverse subtypes of voltage-gated sodium channels (VGSCs) have been found throughout tissues of the brain, muscles and the heart. Neurotoxins extracted from the venom of the Asian scorpion Buthus martensi Karsch (BmK) act as sodium channel-specific modulators and have therefore been widely used to study VGSCs. $\alpha$-type neurotoxins, named BmK I, BmK alV and BmK abT, bind to receptor site-3 on VGSCs and can strongly prolong the inactivation phase of VGSCs. In contrast, $\beta$-type neurotoxins, named BmK AS, BmK AS-1, BmK IT and BmK IT2, occupy receptor site-4 on VGSCs and can suppress peak currents and hyperpolarize the activation kinetics of sodium channels. Accumulating evidence from binding assays of scorpion neurotoxins on VGSCs, however, indicate that pharmacological sensitivity of VGSC subtypes to different modulators is much more complex than that suggested by the simple $\alpha$-type and $\beta$-type neurotoxin distinction. Exploring the mechanisms of possible dynamic interactions between site 3-/4-specific modulators and region- and/or speciesspecific subtypes of VGSCs would therefore greatly expand our understanding of the physiological and pharmacological properties of diverse VGSCs. In this review, we discuss the pharmacological and structural diversity of VGSCs as revealed by studies exploring the binding properties and cross-competitive binding of site 3- or site 4-specific modulators in VGSC subtypes in synaptosomes from distinct tissues of diverse species.

KEYWORDS voltage-gated sodium channel, receptor sites, scorpion neurotoxins

\section{INTRODUCTION}

Voltage-gated sodium channels (VGSCs) are key transmembrane proteins responsible for the initiation and propagation of action potentials in excitable cells (Catterall, 2000). VGSC proteins identified to date have the same structural organization-they all contain four homologous domains (DI-DIV), each with six putative transmembrane segments (S1-S6) (Goldin, 2002). Due to high sequence similarity, characterizing or pharmacologically targeting specific members of the large family of VGSCs has proven to be very challenging. Venomous scorpions produce toxins ( $\alpha$-toxins and $\beta$-toxins) capable of selectively targeting different subtypes of VGSCs. $\alpha$-toxins can slow down the inactivation of VGSCs by associating with receptor site-3, whereas $\beta$-toxins enhance the activation process of VGSCs upon binding to receptor site-4 (Couraud et al., 1982). The two classes of scorpion toxins can be further divided into different groups according to their phylogenetic-relationship or isoform-selectivity: a-mammal toxins, $\alpha$-insect toxins, $\alpha$-like toxins, $\beta$-mammal toxins, depressant or excitatory insect-specific $\beta$-toxins, and $\beta$-like toxins (Zuo and Ji, 2004).

At least nine developmentally or functionally distinct voltage-gated sodium channel subtypes, $\mathrm{Na}_{\mathrm{v}} 1.1$ to $\mathrm{Na}_{\mathrm{v}} 1.9$, have been identified by electrophysiological recordings, biochemical analyses and molecular cloning in mammals (Catterall, 1995). $\mathrm{Na}_{\mathrm{v}} 1.4$ is almost exclusively expressed in skeletal muscles (Trimmer et al., 1989). $\mathrm{Na}_{\mathrm{v}} 1.5$ is predominantly expressed in cardiac muscles (Rogart et al., 1989), although its mRNA and currents are also detectable in neonatal and some adult dorsal root ganglion (DRG) neurons at very low levels (Renganathan et al., 2002). Adult central nervous system (CNS) neurons co-express $\mathrm{Na}_{\mathrm{v}} 1.1, \mathrm{Na}_{\mathrm{v}} 1.2$ 
and $\mathrm{Na}_{\mathrm{v}} 1.6$ (Trimmer and Rhodes, 2004). Developmentally mature DRG sensory neurons express combinations of $\mathrm{Na}_{v} 1.1, \mathrm{Na}_{v} 1.6, \mathrm{Na}_{\mathrm{v}} 1.7, \mathrm{Na}_{\mathrm{v}} 1.8$ and $\mathrm{Na}_{\mathrm{v}} 1.9$ (Black et al., 1996; Dib-Hajj et al., 1998; Amaya et al., 2000). $\mathrm{Na}_{v} 1.3$ is expressed predominantly in neonatal neurons and at very low levels in adult neurons (Beckh et al., 1989). $\mathrm{Na}_{\mathrm{v}} 1.2$, one of the predominant VGSC isoforms in the CNS, is also detectable in adult DRG neurons at low levels (Black et al., 1996).

Unlike in mammals, VGSC subtypes in insects display less diversity in that there is only one kind of $\alpha$-subunit in certain insect species. Up to date, although quite a number of insect VGSCs cDNAs have been reported, most of them have not been functionally expressed in vitro (Ramaswami and Tanouye, 1989; Goldin, 2002). Among them, para from Drosophila (temperature-sensitive paralysis phenotypes) was the first to be identified in insects and has been successfully expressed in Xenopus laevis oocytes (Thackeray and Ganetzky, 1994; Loughney et al., 1989; Ramaswami and Tanouye, 1989). More recently, orthologous genes such as Vssc1 (housefly para channel) in Musca domestica (housefly), as well as $\mathrm{BSC} 1$ and $\mathrm{BgNa}_{\mathrm{v}} 1-1$ (formerly para${ }^{C S M A}$ ) from the German cockroach (Blattella germanica) have also been identified (Dong, 1997; Liu et al., 2001) and expressed well in oocytes (Feng et al., 1995; Smith et al., 1997; Warmke et al., 1997; Tan et al., 2002a, 2002b; Soderlund and Knipple, 2003; Tan et al., 2005). Besides, arachnid VGSCs like $\mathrm{BmNa}_{\mathrm{v}} 1$ (Buthus martensi Karsch, $\mathrm{BmK}$ ), OhNa 1 (Ornithoctonus huwena) (Zuo et al., 2006) and $\mathrm{VdNa}_{\mathrm{v}} 1$ (Varroa destructor) (Wang et al., 2003) have evolved as a unique clade in the VGSC superfamily, which may be useful for investigating adaptive insensitivity of the VGSCs towards self-secreted toxins (Zuo et al., 2006).

Long chain neurotoxins (60-76 amino acid residues), produced by Buthus martensi Karsh, a scorpion species widely distributed in north China, Mongolia and Korea, have previously been characterized as specific modulators of VGSCs (Goudet et al., 2002; Ji and Liu, 2008). It was found that these toxins specifically bind to distinct regions on different VGSCs in either overlapped or separated forms, suggesting that binding sites on VGSCs are diverse (Jia et al., 1999; Li and Ji, 2000; Li et al., 2000a, 2000b; Ji et al., 2002; Chai et al., 2006a, 2006b). Also, key residues involved in toxin-channel inter-recognition or subtype-selective patterns were uncovered by site-directed mutagenesis ( $\mathrm{He}$ et al., 2010, 2011). These findings make BmK neurotoxins good candidates for probing the pharmacological and structural diversity of VGSCs in mammals or insects. Additionally, modulators found to target specific VGSCs may have potential applications in therapeutics against pathological conditions or in bio-pesticide development.

\section{RECEPTOR SITES OF SCORPION TOXINS IN VGSCS}

Findings from numerous studies have led to the conclusion that seven or more distinct receptor sites for neurotoxins exist on VGSCs (Cestèle and Catterall, 2000). Scorpion neurotoxins belong to the class of site- 3 and site- 4 modulators according to their binding affinities and pharmacological activities in VGSCs (Zuo and Ji, 2004). The sections below focus on structural features and molecular composition of receptor sites for scorpion neurotoxins on VGSCs.

\section{Receptor site-3}

The position of receptor site-3 was first investigated using photoreactive derivatives of $\alpha$-scorpion toxins and reconstituted rat brain sodium channels (Darbon et al., 1983; Tejedor and Catterall, 1988). It was shown that the extracellular loops between transmembrane segments S5 and S6 in domain I of the $\alpha$-subunit are involved in the formation of receptor site-3 (Tejedor and Catterall, 1985). In addition, several antibodies that recognize the extracellular loops between transmembrane S5 and S6 segments in domains I and IV prevent $\alpha$ scorpion toxin from binding to receptor site-3, further suggesting the involvement of amino acid residues in that region (Thomsen and Catterall, 1989).

Subsequent studies identified key residues participating in $\alpha$-scorpion toxin binding at receptor site- 3 by site-directed mutagenesis. E1613 in the extracellular loop between transmembrane segments S3 and S4 in domain IV of $\mathrm{rNa}_{\mathrm{v}} 1.2$ was identified to be important for $\alpha$-scorpion toxin binding (Rogers et al., 1996). Likewise, the homologous residues D1612 in cardiac $\mathrm{Na}_{v} 1.5$ (Benzinger et al., 1998), D1428 in skeletal muscle $\mathrm{Na}_{\mathrm{v}} 1.4$ (Ma Z., 2000) and D1600 in brain $\mathrm{Na}_{v} 1.6$ (He et al., 2010) were also demonstrated to be essential for $\alpha$-scorpion toxin binding. Additionally, the residues adjacent to D1613, K1617, P1622 (Rogers et al., 1996) and $\mathrm{K} 1432\left(\mathrm{Na}_{\mathrm{v}} 1.4\right)$ (Leipold et al., 2004) were also found to be partially involved in interaction of site-3 scorpion toxins with VGSCs. Furthermore, studies of chimeric VGSCs between BmK I-sensitive $\mathrm{Na}_{1} 1.5$ and $\mathrm{BmK}$ I-insensitive $\mathrm{Na}_{\mathrm{v}} 1.8$ indicate that bulkiness or cavity formed in the binding region of site-3 scorpion toxins due to alteration of residue composition can subtly affect the sensitivity of VGSCs to the toxins, indicating that D1613 within site-3 is not the sole critical residue. Instead, it is likely that the overall static environment or steric conformation of the extracellular loop determined collaboratively by multiple residues accounts for the binding of scorpion toxins (unpublished data, personal communications with Ji YH, Lab Neuropharmacol \& Neurotoxicol, Shanghai University).

\section{Receptor site-4}

The topology of receptor site-4 began to be elucidated with knowledge of the structural elements involved in the activation of VGSCs (Kontis et al., 1997). It was shown that movement of S4 segments is responsible for channel 
activation, and that domain II in the S4 segment (DIIS4) is critically involved in the voltage dependence of activation (Mitrovic et al., 1998).

A single point mutation G845N in DIIS4 has been shown to greatly attenuate the binding affinities of $\mathrm{Na}_{\mathrm{v}} 1.2$ to $\beta$ scorpion toxins and to abolish the hyperpolarized shift in voltagedependent activation of $\mathrm{Na}_{\mathrm{v}} 1.2$ induced by the toxins (Cestèle et al., 1998). This has led to the notion that the $\mathrm{G} 845$ residue is a critical constituent of receptor site-4. However, there are other extracellular linkers and residues out of this region that may also modify the sensitivity of VGSCs to $\beta$ scorpion toxins. Some examples of such residues are: DII S1-S2, DII S3-S4 and DIII SS2-S6 in mammalian VGSCs for $\beta$-mammal and $\beta$ like toxins (Mantegazza and Cestèle, 2005; Cestèle et al., 2006; Leipold et al., 2006; Cohen et al., 2007), DI S5-SS1, DI SS2-S6, DIII SS2-S6, and DIV SS2-S6 in insect VGSCs for depressant anti-insect toxins (Thomsen and Catterall, 1989). Among these, DIII SS2-S6 is considered to be the recognition site with species-selectivity for BmK IT2, a depressant antiinsect toxin, and DII S3b-S4 is considered the functional voltage-trapping site of VGSCs (He et al., 2011).

\section{PH ARMACOLOGICAL ACTIVITY OF BMK NEUROTOXINS IN MAMMALIAN/INSECT VGSCS}

\section{Bioassays}

The pharmacological activities of unique BmK toxins were first assessed by the ability of the toxins to bind VGSCs in plasma preparations (such as brain synaptosomes and insect nerve cord) using ${ }^{125}$-labelling, surface plasmon resonance (SPR) assays and toxin-specific antibodies (Fig. 1) (Jia et al., 1999; Li and Ji, 2000; Li et al., 2000a, 2000b; Jia et al., 2000; Ji et al., 2002; Chai et al., 2006a, 2006b; Zuo et al., 2006).

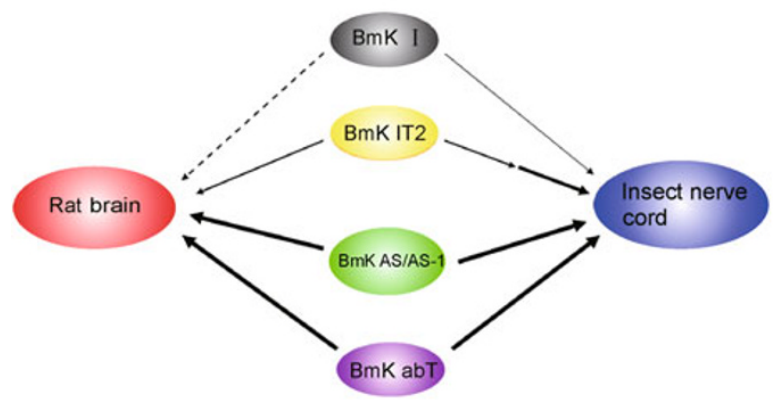

Figure 1. Binding properties of site-3/4 sodium channelspecific modulators to mammalian/insect synaptosomes, where " $\rightarrow$ " indicates specific binding of neurotoxins and “.. toxins. The thickness and number of arrows indicate the extent of binding affinity and number of binding sites of neurotoxins, respectively.
The structure-function relationship of the toxin-channel complex has been studied extensively by site-directed mutagenesis approaches such as Ala scanning and chargereversal/neutralization substitutions. Whereby, the functionally significant residues involved in toxin-channel binding may be identified by assessing the pharmacological capabilities of toxins to the mutated VGSCs through electrophysiological recordings. However, some paradoxes resulting from offset between binding affinity and functional phenotype may often occur due to separate sites responsible for either interrecognition or functional performance in VGSCs, which was witnessed in the case of BmK IT2 whose functional site and recognition site seemed to be separated (Zuo et al., 2006; He et al., 2011).

\section{Binding characteristics of BmK I/BmK a IV/BmK abT}

BmK I, BmK alV and BmK abT act as site-3-specific VGSC modulators and bind to a single class of receptor site in VGSCs in a voltage-dependent manner (Li and Ji, 2000; Chai et al., 2006b).

Similar to classical $\alpha$-toxins, BmK I can inhibit inactivation of VGSCs in crayfish axons (Terakawa et al., 1989; Ji et al., 1994; Ji et al., 1996). However, BmK I can only bind to a single class of non-interacting binding sites with a medium affinity $\left(K_{d}=16.5 \pm 4.4 \mathrm{nmol} / L\right)$ and low binding capacity $\left(B_{\max }\right.$ $=1.05 \pm 0.23 \mathrm{pmol} / \mathrm{mg}$ protein) in cockroach nerve cord synaptosomes, and lacks specific binding in rat brain synaptosomes ( $\mathrm{Li}$ and $\mathrm{Ji}, 2000$ ). This discrepancy can be explained by the possibility that BmK I may interact with mammalian VGSCs with very low affinity, or that most sodium channel subtypes in rat brain synaptosomes are BmK Iinsensitive. The former notion is supported by a subsequent study showing that, at high concentrations $\left(10^{-5} \mathrm{~mol} / \mathrm{L}\right)$, native BmK I could inhibit $\sim 30 \%$ of the specific binding of ${ }^{125} \mathrm{I}$-BmK AS, a distinctive sodium channel site 4 modulator, in rat brain synaptosomes (Li et al., 2000a).

BmK alV was found to have structural identity with both class $\alpha$-toxins and $\alpha$-like toxins. Actually, the structurally transitional molecules are common in the formation process of BmK toxins. Accordingly, BmK alV shares similar binding properties with both classes of toxins that have capabilities of binding to both rat cerebrocortical synaptosomes and cockroach neuronal membranes in a concentration-dependent manner, but could not be dissociated completely after elution. (Chai et al., 2006b).

Another transitional toxin BmK abT, which shares structural identity with class $\beta$ toxin but has similar functions in VGSC with class- $\alpha$ toxins, could bind to rat brain synaptosomes quickly and reached an equilibrium state easily with an association rate constant of about $2.49 \times 10^{6} /(\mathrm{mol} / \mathrm{L} \cdot \mathrm{s})$, five times faster than that for binding to Heliothis nerve cord synaptosomes, for which the association rate constant was about $1.21 \times 10^{7} /(\mathrm{mol} / \mathrm{L} \cdot \mathrm{s})$. By contrast, the dissociation of 
BmK abT from Heliothis synaptosomes (about $0.99 \times 10^{-3} / \mathrm{s}$ ) was about six times faster than that from rat synaptosomes (about $1.57 \times 10^{-3} / \mathrm{s}$ ). The considerable difference in association and dissociation rates suggests that the affinity of mammalian brain sodium channels for BmK abT is higher than that of insect sodium channels. In addition, the binding of BmK abT could be modified by increasing the $\mathrm{K}^{+}$concentration, which strongly indicates that binding of $B m K$ abT to VGSCs in both mammalian brain and insect synaptosomes is dependent on the membrane potential (Ji et al., 2002), as it is with classical $\alpha$-toxins.

Although the binding of BmK alV to the rat cerebrocortical synaptosomes was found to be voltage-dependent (Chai et al., 2006b), that was not the case for binding of the toxin to cockroach neuronal membranes. However, the binding of BmK I to cockroach neuronal membranes was also found to be voltage-independent ( $\mathrm{Li}$ and Ji, 2000).

\section{Binding characteristics of BmK IT2/BmK AS/BmK AS-1}

BmK IT2, BmK AS and BmK AS-1 belong to the class $\beta$ of scorpion neurotoxins, which are defined as site-4-specific VGSC modulators ( $\mathrm{Li}$ and Ji, 2000; Chai et al., 2006b)

$\mathrm{Li}$ et al. found that the specific binding of ${ }^{125} \mathrm{I}-\mathrm{BmK}$ IT2 was undetectable in rat brain synaptosomes (Li et al., 2000b). However, BmK IT2 could suppress the $\mathrm{I}_{\text {peak }}$ of $\mathrm{Na}^{+}$currents in small DRG neurons (Li et al., 2000b; Tan et al., 2001). It is possible that, like BmK I, BmK IT2 binds to the binding sites in mammal brain VGSCs with very low affinity or that very small amounts of BmK IT2-sensitive VGSC subtypes are present in mammalian brain synaptosomes. This speculation is supported by subsequent SPR assays, in which BmK IT2 immobilized on chips was capable of binding to rat brain synaptosomes (Chai et al., 2006a). The difference in the outcomes of the two bioassays examining the binding affinity of BmK IT2 to rat brain synaptosomes was considered to be attributed to the different binding phases they monitored (Chai et al., 2006a; Zuo et al., 2006). In addition, it was demonstrated that BmK IT2 has two non-interacting binding sites in insect neuronal membranes: a high-affinity and low-capacity binding site, as well as a low-affinity and high-capacity binding site ( $\mathrm{Li}$ et al., 2000b), which is consistent with the results of the two bioassays mentioned above.

BmK AS, another site-4 toxin, seemed to bind specifically to a single class of non-interacting binding sites in rat brain and cockroach nerve cord synaptosomes with high affinity and low capacity, respectively. The binding capability of BmK AS was strong and it dissociated partially from the binding sites in both kinds of synaptosomes in a voltage-independent manner (Li et al., 2000a). BmK AS-1 shares almost identical binding features and amino acid sequence with BmK AS (Jia et al., 1999) and thus belongs to the same clade as BmK AS.

\section{Binding characteristics of BmK neurotoxins with two arachnid VGSCs}

Scorpion toxins lack any effects on arachnid VGSCs (Terakawa et al., 1989; Legros et al., 1998). One possible explanation for this is that the binding affinities of the toxins to scorpion sodium channels are diminished. Zuo et al. conducted SPR bioassay experiments and found that synaptosomes from BmK scorpion or those from the spider Ornithoctonus huwena could rapidly bind to chips loaded with BmK AS or BmK IT2 in a concentration-dependent manner, but scorpion synaptosomes had much weaker affinity than spider synaptosomes (Zuo et al., 2006). Notably, neither scorpion synaptosomes nor spider synaptosomes could bind to chips loaded with BmK I. Although the lack of binding affinity of BmK I to scorpion synaptosomes seems to be consistent with the known adaptive insensitivity of scorpion VGSCs towards their own toxins, it is unclear whether an appropriate static environment required for BmK I binding to VGSCs was created by shielding during the toxin immobilization process (Zuo et al., 2006). These speculation should therefore be interpreted with some caution.

\section{DIVERSITY OF RECEPTOR SITES IN MAMMALIAN/ INSECT VGSCS PROBED BY BMK NEUROTOXINS}

The functional and pharmacological properties of VGSCs have been studied by conducting electrophysiological recordings in various tissues and compared across many species (Mandel, 1992). The highly specific expression of various VGSC subtypes in distinct tissues raises interesting questions about the function of VGSCs. One possibility is that structural diversity of VGSCs may play an indispensable role in neuronal signal decoding within certain tissues.

The receptor sites at which scorpion neurotoxins bind and affect channel gating have been found to be allosterically coupled, suggesting that conformational changes of the gating motif can alter the equilibrium between the open and the closed/inactivated states of sodium channels and binding affinity of toxins at receptor sites (De Lima et al., 2007). For this reason, the section below will discuss how competitive binding assay was used to qualitatively understand the bilateral relationship between VGSCs and BmK neurotoxins, and to identify the binding sites of toxins on VGSCs.

\section{Competitive binding properties of BmK neurotoxins in insect VGSCs}

All scorpion neurotoxins are toxic to insects and can bind to intact insect nerve cord, which is not surprising given that scorpions are the natural predators of these insect species.

Both radio-labelling and SPR assays have demonstrated that either native $\mathrm{BmK}$ I or other types of scorpion toxins are 
capable of inhibiting the binding of BmK I to cockroach neuronal membranes. However, the inhibitory capability of BmK IT2 or BmK IT seems to be much weaker than that of BmK AS and BmK AS-1. In contrast, BmK AS and BmK IT2 can directly bind to insect VGSCs with high affinity, while native $\mathrm{BmK}$ I is incapable of inhibiting the binding of $\mathrm{BmK}$ AS to insect VGSCs (Jia et al., 1999; Li and Ji, 2000). These results suggest that the inhibitory effects of either excitatory (BmK IT) or depressant (BmK IT2) insect-selective toxins on the binding of $\alpha$-like toxins (BmK I) to insect VGSCs can be attributed to allosteric modulation in VGSCs, as seen in $\beta$ type scorpion toxins (Gordon et al., 1998), which leads to a decrease in BmK I affinity (Cestèle et al., 1998). However, it cannot be ruled out whether the receptor sites of $\alpha$-like scorpion toxins on insect VGSCs partially overlap with those of excitatory and depressant insect-selective toxins and BmK AS-type toxins.

The specific binding of BmK IT2 with VGSCs on cockroach nerve membranes can be completely inhibited by native BmK IT2, BmK AS and BmK AS-1 with two different inhibition constants, which supports the concept of two binding sites of BmK IT2 in insect synaptosomes, one with high affinity and low capacity, and the other with low affinity and high capacity (Li et al., 2000b; Chai et al., 2006a). However, it is noteworthy that there may be only one binding site on the sodium channel. The other binding site may be on synaptosomal membranes, as is the case for LqhIT2, which is an anti-insect depressant $\beta$-toxin that shares $>80 \%$ structural identity with BmK IT2 (Cohen et al., 2007). For the reason that depressant toxins can completely inhibit the binding of excitatory toxins and excitatory toxins have been found to compete only for the high-affinity binding site of depressant toxins (Cestèle et al., 1997; He et al., 2011), the receptor site for BmK IT2 on insect VGSCs may be exactly the high-affinity binding site (Zuo and Ji, 2004). Furthermore, considering the complete inhibition of BmK IT2 binding to insect synaptosomes by BmK AS/AS-1 and the high degree of sequence similarity among them ( $\mathrm{Ji}$ et al., 1995), the binding sites of BmK AS and BmK AS-1 might partially overlap with the high-affinity binding site of BmK IT2 on insect VGSCs (Li et al., 2000b). Additionally, it is surprising that only about $20 \%$ of the specific binding of BmK IT2 to cockroach nerve cord synaptosomes was found to be depressed at high concentrations of BmK I ( $\mathrm{Li}$ et al., 2000b). These observations lend support to the notion that receptor site-3, which is recognized by sea anemone toxin, and $\alpha$-like and $\alpha$-type insect scorpion toxins, does not overlap with but is closely related to the binding sites of depressantinsect toxins (such as BmK IT2) on insect VGSCs (Cestèle et al., 1997).

BmK abT, a novel transitional neurotoxic polypeptide that lies between $\alpha$ and $\beta$ subgroups in the phylogenetic tree (Zuo and Ji, 2004), was found to completely displace the binding of BmK IT2 to cockroach neuronal membranes (Chai et al., 2006a). Conversely, BmK IT2 could also significantly inhibit the binding of BmK abT to Heliothis nerve cord synaptosomes (Ji et al., 2002). The binding site of BmK abT is thus thought to be close to that of BmK IT2 on insect sodium channels.

Competitive inhibition of BmK alV binding to cockroach neuronal membranes by BmK AS, BmK IT2 and BmK abT indicates that the receptor site of BmK alV on insect VGSCs might be shared, at least in part, with the putative receptor site-3 of $\beta$-like neurotoxins (Chai et al., 2006b).

\section{Competitive binding properties of BmK neurotoxins on mammalian VGSCs}

The binding affinity of BmK neurotoxins in mammalian brain synaptosomes appears to be lower compared to their binding in insect nerve cords (Li et al., 2000b; Li and Ji, 2000; Chai et al., 2006a). Structural alterations in conserved regions within receptor sites may account for such divergence in pharmacological sensitivity to the same toxin.

Similar to the findings in insect nerve cords, it has been found that specific binding of BmK IT2 to rat cerebrocortical and hippocampal synaptosomes could also be significantly inhibited by BmK AS (Chai et al., 2006a). Likewise, the specific binding of BmK AS could be significantly inhibited by native BmK AS, BmK AS-1, BmK IT2 and BmK IT in synaptosomes from both brain areas ( $\mathrm{Li}$ et al., 2000a). Unexpectedly, the binding of BmK AS in mammalian synaptosomes was found to be inhibited by BmK I at $10^{-5}-10^{-9} \mathrm{~mol} / \mathrm{L}$ by only about $20 \%-30 \%$, and the binding in insect synaptosomes was unaffected (Jia et al., 1999). Furthermore, the observations that BmK AS-1-specific antibody could fully cross-react with BmK AS, partially crossreact with BmK IT2, but had no effect on BmK I and BmK IT (Jia et al., 2000) further support the notion that BmK IT2 and BmK AS/AS-1 may share closely related binding sites on mammal brain-type and insect VGSCs, and may not share binding sites with BmK I or BmK IT.

The binding site of $B m K$ abT appears to be unrelated to that of BmK AS, BmK IT2 or BmK I. BmK IT2 has been found to be capable of slightly inhibiting the binding of $B m K$ abT to rat cerebrocortical synaptosomes (Ji et al., 2002). Given the remarkable ability of BmK IT2 to inhibit the binding of BmK $a b T$ to insect nerve cords, the binding site of BmK abT is considered to overlap partially with that of BmK IT2 on mammalian brain-type VGSCs but completely with that of BmK IT2 on insect VGSCs.

Interestingly, BmK aIV was found to be partially capable of inhibiting BmK IT2 binding to cockroach and rat cerebrocortical synaptosomes, but not to rat hippocampal synaptosomes, indicating that the receptor sites of BmK IT2 and BmK alV have subtle overlaps in subtypes of VGSCs distributed in insect, rat cerebrocortical and hippocampal neuronal membranes (Chai et al., 2006a). 

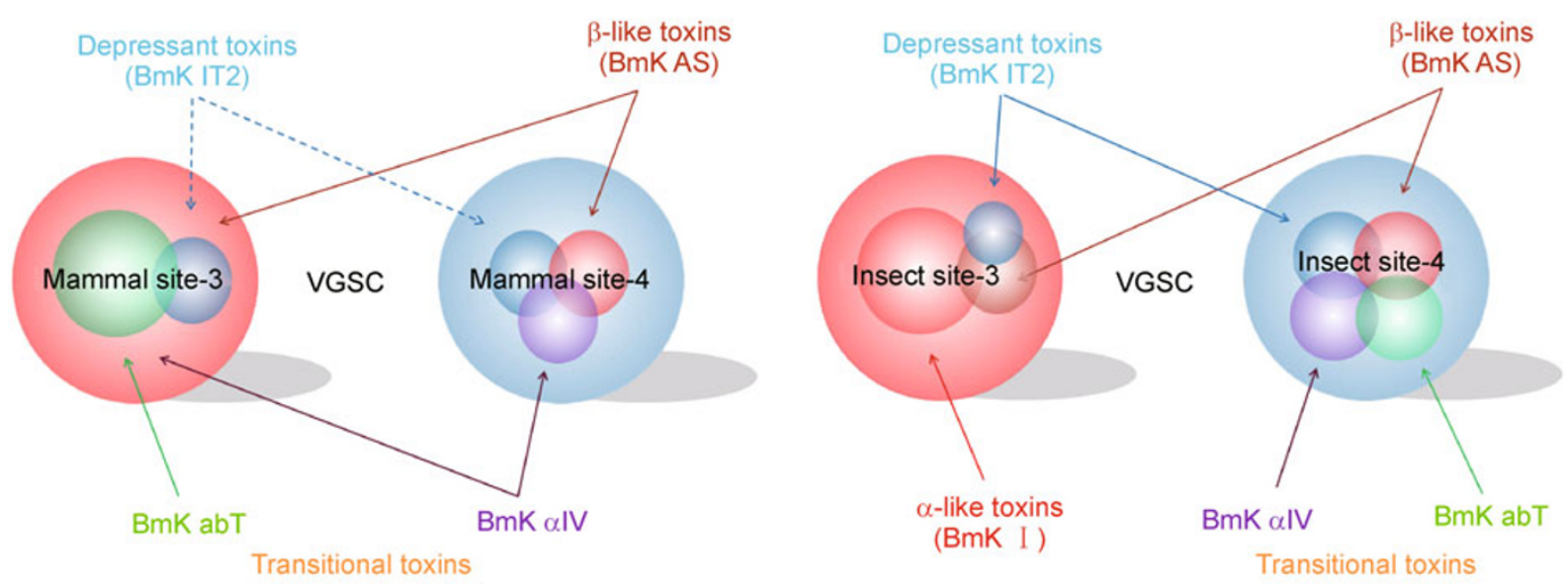

Figure 2. Distinct binding sites of site-3/4 sodium channel-specific modulators on mammalian/insect sodium channels.

\section{CONCLUDING REMARKS}

Structurally and functionally diverse VGSC subtypes play critical roles in the initiation, maintenance and conduction of excitatory signals with high fidelity in the nervous system. Due to their vital importance in neuronal physiology and their involvement in channelopathies like pain, epilepsy, and arrhythmia, proper understanding of VGSCs and their pharmacological activators and depressants is vital for the development of novel therapies.

As sodium channel-specific modulators, BmK neurotoxins have great potentials for allowing us to probe the intriguing subtle variations in VGSCs found across different tissues and species. More detailed investigations into the dynamic toxinchannel interactions, the conformational alterations and chemical-shift related to interactive residues in VGSCs are needed. The availability of unique BmK neurotoxins against specific VGSCs will likely help us tremendously in elucidating the pharmacological and structural diversity of VGSCs (Fig. 2).

\section{ACKNOWLEDGEMENTS}

This study was supported by the National Basic Research Program of China (Grant Nos. 1999054001, 2006CB500801, and 2010CB529806), partially by grants from Key discipline "Molecular Physiology" of Shanghai Education Committee. This work is dedicated to the third author's beloved student Dr. Zhifang Chai who made important contributions to the binding assays and recently passed away due to severe lymph cancer at a young age.

\section{REFERENCES}

Amaya, F., Decosterd, I., Samad, T.A., Plumpton, C., Tate, S., Mannion, R.J., Costigan, M., and Woolf, C.J. (2000). Diversity of expression of the sensory neuron-specific TTX-resistant voltagegated sodium ion channels SNS and SNS2. Mol Cell Neurosci 15, 331-342.
Beckh, S., Noda, M., Lübbert, H., and Numa, S. (1989). Differential regulation of three sodium channel messenger RNAs in the rat central nervous system during development. EMBO J 8, 3611-3616.

Benzinger, G.R., Kyle, J.W., Blumenthal, K.M., and Hanck, D.A. (1998). A specific interaction between the cardiac sodium channel and site-3 toxin anthopleurin B. J Biol Chem 273, 80-84.

Black, J.A., Dib-Hajj, S., McNabola, K., Jeste, S., Rizzo, M.A., Kocsis, J.D., and Waxman, S.G. (1996). Spinal sensory neurons express multiple sodium channel alpha-subunit mRNAs. Brain Res Mol Brain Res 43, 117-131.

Catterall, W.A. (1995). Structure and function of voltage-gated ion channels. Annu Rev Biochem 64, 493-531.

Catterall, W.A. (2000). From ionic currents to molecular mechanisms: the structure and function of voltage-gated sodium channels. Neuron 26, 13-25.

Cestèle, S., and Catterall, W.A. (2000). Molecular mechanisms of neurotoxin action on voltage-gated sodium channels. Biochimie 82, 883-892.

Cestèle, S., Kopeyan, C., Oughideni, R., Mansuelle, P., Granier, C., and Rochat, H. (1997). Biochemical and pharmacological characterization of a depressant insect toxin from the venom of the scorpion Buthacus arenicola. Eur J Biochem 243, 93-99.

Cestèle, S., Qu, Y., Rogers, J.C., Rochat, H., Scheuer, T., and Catterall, W.A. (1998). Voltage sensor-trapping: enhanced activation of sodium channels by beta-scorpion toxin bound to the S3-S4 loop in domain II. Neuron 21, 919-931.

Cestèle, S., Yarov-Yarovoy, V., Qu, Y., Sampieri, F., Scheuer, T., and Catterall, W.A. (2006). Structure and function of the voltage sensor of sodium channels probed by a beta-scorpion toxin. J Biol Chem 281, 21332-21344.

Chai, Z.F., Bai, Z.T., Liu, T., Pang, X.Y., and Ji, Y.H. (2006a). The binding of BmK IT2 on mammal and insect sodium channels by surface plasmon resonance assay. Pharmacol Res 54, 85-90.

Chai, Z.F., Zhu, M.M., Bai, Z.T., Liu, T., Tan, M., Pang, X.Y., and Ji, Y. H. (2006b). Chinese-scorpion (Buthus martensi Karsch) toxin BmK alphalV, a novel modulator of sodium channels: from genomic organization to functional analysis. Biochem J 399, 445- 453.

Cohen, L., Ilan, N., Gur, M., Stühmer, W., Gordon, D., and Gurevitz, 
M. (2007). Design of a specific activator for skeletal muscle sodium channels uncovers channel architecture. J Biol Chem 282, 29424-29430.

Couraud, F., Jover, E., Dubois, J.M., and Rochat, H. (1982). Two types of scorpion receptor sites, one related to the activation, the other to the inactivation of the action potential sodium channel. Toxicon 20, 9-16.

Darbon, H., Jover, E., Couraud, F., and Rochat, H. (1983). Photoaffinity labeling of alpha- and beta- scorpion toxin receptors associated with rat brain sodium channel. Biochem Biophys Res Commun 115, 415-422.

De Lima, M.E., Figueiredo, S.G., Pimenta, A.M., Santos, D.M., Borges, M.H., Cordeiro, M.N., Richardson, M., Oliveira, L.C., Stankiewicz, M., and Pelhate, M. (2007). Peptides of arachnid venoms with insecticidal activity targeting sodium channels. Comp Biochem Physiol C Toxicol Pharmacol 146, 264-279.

Dib-Hajj, S.D., Black, J.A., Cummins, T.R., Kenney, A.M., Kocsis, J. D., and Waxman, S.G. (1998). Rescue of alpha-SNS sodium channel expression in small dorsal root ganglion neurons after axotomy by nerve growth factor in vivo. J Neurophysiol 79, 2668-2676.

Dong, K. (1997). A single amino acid change in the para sodium channel protein is associated with knockdown-resistance (kdr) to pyrethroid insecticides in German cockroach. Insect Biochem Mol Biol 27, 93-100.

Feng, G., Deák, P., Chopra, M., and Hall, L.M. (1995). Cloning and functional analysis of TipE, a novel membrane protein that enhances Drosophila para sodium channel function. Cell 82, $1001-1011$.

Goldin, A.L. (2002). Evolution of voltage-gated $\mathrm{Na}(+)$ channels. J Exp Biol 205, 575-584.

Gordon, D., Savarin, P., Gurevitz, M., and Zinn-Justin, S. (1998). Functional anatomy of scorpion toxins affecting sodium channels. J Toxicol Toxin Rev 2, 131-159.

Goudet, C., Chi, C.W., and Tytgat, J. (2002). An overview of toxins and genes from the venom of the Asian scorpion Buthus martensi Karsch. Toxicon 40, 1239-1258.

He, H., Liu, Z., Dong, B., Zhang, J., Shu, X., Zhou, J., and Ji, Y. (2011). Localization of receptor site on insect sodium channel for depressant $\beta$-toxin BmK IT2. PLoS ONE 6, e14510.

He, H., Liu, Z., Dong, B., Zhou, J., Zhu, H., and Ji, Y. (2010). Molecular determination of selectivity of the site 3 modulator (BmK I) to sodium channels in the CNS: a clue to the importance of Nav1.6 in BmK I-induced neuronal hyperexcitability. Biochem J 431, 289-298.

Ji, Y.H., Li, Y.J., Zhang, J.W., Song, B.L., Yamaki, T., Mochizuki, T., Hoshino, M., and Yanaihara, N. (1999). Covalent structures of BmK AS and BmK AS-1, two novel bioactive polypeptides purified from Chinese scorpion Buthus martensi Karsch. Toxicon 37, 519-536.

Ji, Y.H., and Liu, T. (2008). The study of sodium channels involved in pain responses using specific modulators. Sheng Li Xue Bao 60, 628-634.

Ji, Y.H., Mansuelle, P., Terakawa, S., Kopeyan, C., Yanaihara, N., Hsu, K., and Rochat, H. (1996). Two neurotoxins (BmK I and BmK II) from the venom of the scorpion Buthus martensi Karsch: purification, amino acid sequences and assessment of specific activity. Toxicon 34, 987-1001.
Ji, Y.H., Mansuelle, P., Xu, K., Granier, C., Kopeyan, C., Terakawa, S., and Rochat, H. (1994). Amino acid sequence of an excitatory insect-selective toxin (BmK IT) from venom of the scorpion Buthus martensi Karsch. Sci China B 37, 42-49.

Ji, Y.H., Wang, W.X., Wang, Q., and Huang, Y.P. (2002). The binding of BmK abT, a unique neurotoxin, to mammal brain and insect $\mathrm{Na}$ (+) channels using biosensor. Eur J Pharmacol 454, 25-30.

Jia, L.Y., Xie, H.F., and Ji, Y.H. (2000). Characterization of four distinct monoclonal antibodies specific to BmK AS-1, a novel scorpion bioactive polypeptide. Toxicon 38, 605-617.

Jia, L.Y., Zhang, J.W., and Ji, Y.H. (1999). Biosensor binding assay of BmK AS-1, a novel $\mathrm{Na}+$ channel-blocking scorpion ligand on rat brain synaptosomes. Neuroreport 10, 3359-3362.

Kontis, K.J., Rounaghi, A., and Goldin, A.L. (1997). Sodium channel activation gating is affected by substitutions of voltage sensor positive charges in all four domains. J Gen Physiol 110, 391-401.

Legros, C., Martin-Eauclaire, M.F., and Cattaert, D. (1998). The myth of scorpion suicide: are scorpions insensitive to their own venom? J Exp Biol 201, 2625-2636.

Leipold, E., Hansel, A., Borges, A., and Heinemann, S.H. (2006). Subtype specificity of scorpion beta-toxin Tz1 interaction with voltage-gated sodium channels is determined by the pore loop of domain 3. Mol Pharmacol 70, 340-347.

Leipold, E., Lu, S., Gordon, D., Hansel, A., and Heinemann, S.H. (2004). Combinatorial interaction of scorpion toxins Lqh-2, Lqh-3, and LqhalphalT with sodium channel receptor sites-3. Mol Pharmacol 65, 685-691.

Li, Y.J., and Ji, Y.H. (2000). Binding characteristics of BmK I, an alphalike scorpion neurotoxic polypeptide, on cockroach nerve cord synaptosomes. J Pept Res 56, 195-200.

Li, Y.J., Liu, Y., and Ji, Y.H. (2000a). BmK AS: new scorpion neurotoxin binds to distinct receptor sites of mammal and insect voltage-gated sodium channels. J Neurosci Res 61, 541-548.

Li, Y.J., Tan, Z.Y., and Ji, Y.H. (2000b). The binding of BmK IT2, a depressant insect-selective scorpion toxin on mammal and insect sodium channels. Neurosci Res 38, 257-264.

Liu, Z., Chung, I., and Dong, K. (2001). Alternative splicing of the BSC1 gene generates tissue-specific isoforms in the German cockroach. Insect Biochem Mol Biol 31, 703-713.

Loughney, K., Kreber, R., and Ganetzky, B. (1989). Molecular analysis of the para locus, a sodium channel gene in Drosophila. Cell 58, 1143-1154.

Ma Z., T. L., Lu S., Kong J., Gordon D., Kallen R.G., (2000). The domain 4 S3-S4 extracellular loop provides molecular determinants for binding of -scorpion toxins (Lqhll, and LqhIT) to the voltage-gated rat skeletal muscle $\mathrm{Na}+$ channel (rSkM1). Biophys Soc Abstract.

Mandel, G. (1992). Tissue-specific expression of the voltagesensitive sodium channel. J Membr Biol 125, 193-205.

Mantegazza, M., and Cestèle, S. (2005). Beta-scorpion toxin effects suggest electrostatic interactions in domain II of voltage-dependent sodium channels. J Physiol 568, 13-30.

Mitrovic, N., George, A.L. Jr, and Horn, R. (1998). Independent versus coupled inactivation in sodium channels. Role of the domain $2 \mathrm{~S} 4$ segment. J Gen Physiol 111, 451-462.

Ramaswami, M., and Tanouye, M.A. (1989). Two sodium-channel genes in Drosophila: implications for channel diversity. Proc Natl Acad Sci USA 86, 2079-2082. 
Renganathan, M., Dib-Hajj, S., and Waxman, S.G. (2002). Na(v)1.5 underlies the 'third TTX-R sodium current' in rat small DRG neurons. Brain Res Mol Brain Res 106, 70-82.

Rogart, R.B., Cribbs, L.L., Muglia, L.K., Kephart, D.D., and Kaiser, M. W. (1989). Molecular cloning of a putative tetrodotoxin-resistant rat heart $\mathrm{Na}+$ channel isoform. Proc Natl Acad Sci USA 86, 8170-8174.

Rogers, J.C., Qu, Y., Tanada, T.N., Scheuer, T., and Catterall, W.A. (1996). Molecular determinants of high affinity binding of alphascorpion toxin and sea anemone toxin in the S3-S4 extracellular loop in domain IV of the $\mathrm{Na}+$ channel alpha subunit. J Biol Chem 271, 15950-15962.

Smith, T.J., Lee, S.H., Ingles, P.J., Knipple, D.C., and Soderlund, D.M. (1997). The L1014F point mutation in the house fly Vssc1 sodium channel confers knockdown resistance to pyrethroids. Insect Biochem Mol Biol 27, 807-812.

Soderlund, D.M., and Knipple, D.C. (2003). The molecular biology of knockdown resistance to pyrethroid insecticides. Insect Biochem Mol Biol 33, 563-577.

Tan, J., Liu, Z., Nomura, Y., Goldin, A.L., and Dong, K. (2002a). Alternative splicing of an insect sodium channel gene generates pharmacologically distinct sodium channels. J Neurosci 22, 5300-5309.

Tan, J., Liu, Z., Tsai, T.D., Valles, S.M., Goldin, A.L., and Dong, K. (2002b). Novel sodium channel gene mutations in Blattella germanica reduce the sensitivity of expressed channels to deltamethrin. Insect Biochem Mol Biol 32, 445- 454.

Tan, J., Liu, Z., Wang, R., Huang, Z.Y., Chen, A.C., Gurevitz, M., and Dong, K. (2005). Identification of amino acid residues in the insect sodium channel critical for pyrethroid binding. Mol Pharmacol 67 , 513-522.

Tan, Z.Y., Xiao, H., Mao, X., Wang, C.Y., Zhao, Z.Q., and Ji, Y.H. (2001). The inhibitory effects of BmK IT2, a scorpion neurotoxin on rat nociceptive flexion reflex and a possible mechanism for modulating voltage-gated $\mathrm{Na}(+)$ channels. Neuropharmacology
40, 352-357.

Tejedor, F.J., and Catterall, W.A. (1988). Site of covalent attachment of alpha-scorpion toxin derivatives in domain I of the sodium channel alpha subunit. Proc Natl Acad Sci USA 85, 8742-8746.

Terakawa, S., Kimura, Y., Hsu, K., and Ji, Y.H. (1989). Lack of effect of a neurotoxin from the scorpion Buthus martensi Karsch on nerve fibers of this scorpion. Toxicon 27, 569-578.

Thackeray, J.R., and Ganetzky, B. (1994). Developmentally regulated alternative splicing generates a complex array of Drosophila para sodium channel isoforms. J Neurosci 14, 2569-2578.

Thomsen, W.J., and Catterall, W.A. (1989). Localization of the receptor site for alpha-scorpion toxins by antibody mapping: implications for sodium channel topology. Proc Natl Acad Sci USA 86, 10161-10165.

Trimmer, J.S., Cooperman, S.S., Tomiko, S.A., Zhou, J.Y., Crean, S. M., Boyle, M.B., Kallen, R.G., Sheng, Z.H., Barchi, R.L., Sigworth, F.J.,et al. (1989). Primary structure and functional expression of a mammalian skeletal muscle sodium channel. Neuron 3, 33-49.

Trimmer, J.S., and Rhodes, K.J. (2004). Localization of voltage-gated ion channels in mammalian brain. Annu Rev Physiol 66, 477-519.

Wang, R., Huang, Z.Y., and Dong, K. (2003). Molecular characterization of an arachnid sodium channel gene from the varroa mite (Varroa destructor). Insect Biochem Mol Biol 33, 733-739.

Warmke, J.W., Reenan, R.A., Wang, P., Qian, S., Arena, J.P., Wang, J., Wunderler, D., Liu, K., Kaczorowski, G.J., Van der Ploeg, L.H., et al.(1997). Functional expression of Drosophila para sodium channels. Modulation by the membrane protein TipE and toxin pharmacology. J Gen Physiol 110, 119-133.

Zuo, X.P., He, H.Q., He, M., Liu, Z.R., Xu, Q., Ye, J.G., and Ji, Y.H. (2006). Comparative pharmacology and cloning of two novel arachnid sodium channels: Exploring the adaptive insensitivity of scorpion to its toxins. FEBS Lett 580, 4508-4514.

Zuo, X.P., and Ji, Y.H. (2004). Molecular mechanism of scorpion neurotoxins acting on sodium channels: insight into their diverse selectivity. Mol Neurobiol 30, 265-278. 